Pacific

Journal of

Mathematics

\title{
DISTANCE AND BRIDGE POSITION
}

DAVID BACHMAN AND SAUL SCHLEIMER 


\title{
DISTANCE AND BRIDGE POSITION
}

\author{
DAVID BACHMAN AND SAUL SCHLEIMER
}

\begin{abstract}
J. Hempel's definition of the distance of a Heegaard surface generalizes to a notion of complexity for any knot that is in bridge position with respect to a Heegaard surface. Our main result is that the distance of a knot in bridge position is bounded above by twice the genus, plus the number of boundary components, of an essential surface in the knot complement. As a consequence knots constructed via sufficiently high powers of pseudoAnosov maps have minimal bridge presentations which are thin.
\end{abstract}

\section{Introduction}

Hempel's definition [2001] of the distance of a Heegaard splitting is a natural measure of complexity, generalizing the standard notions of reducibility (distance zero), weak reducibility (distance at most one), and strong irreducibility (distance at least two). Hempel proves that there exist Heegaard splittings of arbitrarily high distance.

In his Ph.D. thesis, K. Hartshorn related the distance of a Heegaard splitting to the genus of any essential surface, thus refining work of T. Kobayashi [1988]:

Theorem [Hartshorn 1999]. Let $M$ be a closed, orientable, irreducible 3-manifold with Heegaard splitting $F$. Suppose $M$ contains an orientable, incompressible surface $S$. Then the distance of $F$ is bounded above by twice the genus of $S$.

We introduce our results by recalling a generalization of the curve complex for surfaces with nonempty boundary. This allows us to translate Hempel's definition of distance for Heegaard splittings to a definition of distance for knots that are in bridge position with respect to a Heegaard surface [Morimoto and Sakuma 1991]. Our main result is a translation of Hartshorn's Theorem into this new context:

Theorem 5.1. Let $K$ be a knot in a closed, orientable 3-manifold $M$ which is in bridge position with respect to a Heegaard surface $F$. Let $S$ be a properly embedded, orientable, essential surface in $M_{K}$. Then the distance of $K$ with respect to $F$ is bounded above by twice the genus of $S$ plus $|\partial S|$.

MSC2000: 57M25, 57M27.

Keywords: Heegaard splitting, curve complex. 
In the special case of a meridional disk we find that a stronger result holds; the distance of $K$ with respect to $F$ is zero. This follows from a variant of the Haken Lemma (see Lemma 4.1).

Although our proof contains Hartshorn's result as a special case $(K=\varnothing)$, there is an interesting qualitative difference. Unlike Hartshorn, we make no minimality assumption on the way in which $S$ intersects $F$. That is, any generic position of $S$ with respect to $F$ forces the bound on distance as stated in the theorem.

The main idea behind our proof is to simply count saddles. Let $d(K, F)$ denote the distance of $K$ with respect to $F$. It is a standard technique in 3-manifold topology to use a Heegaard splitting $F$ for a 3-manifold $M$ to define a height function $h$ on $M$. This, in turn, induces a height function on a surface $S$ in $M$. With respect to this height function $S$ will have maxima, minima, and saddles. The moral of the story is that each critical point of $S$ either

(1) contributes at most 1 to $d(K, F)$ and exactly -1 to the Euler characteristic of $S$, or

(2) contributes nothing to $d(K, F)$ and nothing to the Euler characteristic of $S$.

Hence, the distance of $K$ with respect to $F$ would then be bounded by the negative of the Euler characteristic of $S$. Unfortunately, for Heegaard splittings the above classification isn't exactly correct. We find that there may be at most two special critical points that each contribute one to the distance of $K$, but nothing to the Euler characteristic of $S$. This gives the bound

$$
d(K, F) \leq-\chi(S)+2=2 g(S)+|\partial S| .
$$

We note that several authors have explicitly computed the distances of various classes of knots (using varying definitions of distance). See, for example, [Akiyoshi et al. 2000; Morimoto 1989; Saito 2004].

In the final section we present corollaries to Theorem 5.1. Among these are:

Corollary 6.1. Suppose $K$ is a knot in $S^{3}$ whose distance is $d(K, F)$ with respect to a bridge sphere $F$. Then the genus of $K$ is at least $\frac{1}{2}(d(K, F)-1)$.

Corollary 6.2. If $K$ is a knot whose distance is at least 3 with respect to some Heegaard surface, the complement of $K$ is hyperbolic.

Finally, we define the bridge link associated to an element of the braid group $B_{2 n}$ to be the link obtained by gluing two trivial $n$-strand tangles by this element. By a construction essentially due to Kobayashi [1988], powers of certain pseudoAnosov maps give associated bridge links with arbitrarily high distance. Suppose $\phi$ is such a map. Then it follows from Corollary 6.5 that for all sufficiently high powers of $\phi$ if the associated link is a knot, its minimal bridge presentation is thin. 
A priori, bridge knots associated to high powers of pseudo-Anosov maps might have low bridge numbers. We conjecture that this is not in fact possible:

Conjecture. Suppose $K$ is a knot whose distance is at least 2 with respect to some Heegaard surface $F$. Then the distance of $K$ with respect to any other Heegaard surface is bounded above by $\chi(F-K)+2$.

Compare this to the statement of Theorem 5.1. In the theorem we assert that the distance of a knot with respect to a Heegaard surface is bounded by two plus the Euler characteristic of an essential surface. In the conjecture we propose that distance is similarly bounded by a strongly irreducible surface.

\section{Basic definitions}

In this section we give the definitions that will be used throughout the paper. Let $K$ be a knot in a closed, orientable 3-manifold, $M$. Let $M_{K}=M-N(K)$ where $N(K)$ denotes a regular neighborhood of $K$. For the remainder of this paper all surfaces $S$ in $M_{K}$ will be embedded, compact, and orientable with $S \cap \partial M_{K} \subset \partial S$.

Definition 2.1. A cut surface (see Figure 1) is either

(1) a disk $E \subset M_{K}$ such that $E \cap \partial M_{K}=\varnothing$,

(2) a bigon $E \subset M_{K}$ such that $E \cap \partial M_{K}$ is an arc, or

(3) an annulus $E \subset M_{K}$ with exactly one meridional boundary component on $\partial M_{K}$. In other words, $E \cap \partial M_{K}$ is a loop bounding a disk in $\overline{N(K)}$.

If $E$ is a cut surface and $\gamma=\overline{\partial E-\partial M_{K}}$ we say that $\gamma$ bounds a cut surface.
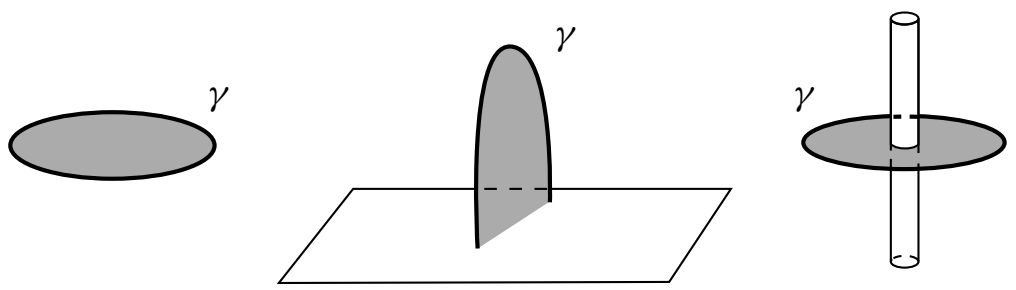

Figure 1. Disk, bigon, and meridional cut surfaces.

A properly embedded simple curve in $S$ is inessential if it bounds a subsurface of $S$ that is a cut surface, and essential otherwise.

Suppose $\gamma$ bounds a cut surface $E$, that $S$ is properly embedded in $M_{K}$, and that $S \cap E=\gamma$. We may then surger $S$ along $E$ by replacing a neighborhood of $\gamma$ in $S$ with two parallel copies of $E$. If $\gamma$ is essential in $S$ we say $E$ is a compression for $S$. In this case we also say $\gamma$ bounds a compression for $S$. 
A properly embedded surface $S \subset M_{K}$ is essential if first there are no curves on $S$ which bound compressions in $M_{K}$ and second $\partial S$ (if nonempty) is not nullhomotopic on $\partial M_{K}$. We also consider a 2-sphere to be essential if it does not bound a ball in $M_{K}$. This notion of essentialness is not identical to that of "superincompressible" found in [Morgan and Bass 1984].

A handlebody is a 3-manifold homeomorphic to the closure of a regular neighborhood of a compact, connected graph in $\mathbb{R}^{3}$. If such a graph has no valence-one vertices and the corresponding handlebody has nonzero genus, the graph's image under such a homeomorphism is a spine of the handlebody. We will insist that the spine of a 3-ball be a single edge.

A closed surface $F$ in $M$ is a Heegaard surface of $\mathrm{M}$ if $F$ separates $M$ into two handlebodies. An arc properly embedded in $H$ is trivial if it bounds a bigon in $H$. Suppose $K$ is a knot in a 3-manifold $M$ with Heegaard surface $F$. The knot $K$ is in bridge position with respect to $F$ [Morimoto and Sakuma 1991] if $K$ meets each of the handlebodies bounded by $F$ in a collection of trivial arcs. Such a position is sometimes referred to as a $(g, b)$-presentation of $K$, where $g=\operatorname{genus}(F)$ and $2 b=|K \cap F|$.

\section{The arc complex}

Following Hempel's definition [2001] of the distance of a Heegaard splitting, we now define the distance of a knot $K$ that is in bridge position with respect to a Heegaard surface $F \subset M$. Set

$$
M_{K}=M-N(K) \quad \text { and } \quad F_{K}=F \cap M_{K} .
$$

Construct a 1-complex $\Gamma\left(F_{K}\right)$ as follows: for each proper isotopy class of essential curves in $F_{K}$ there is a vertex of $\Gamma\left(F_{K}\right)$. There is an edge of $\Gamma\left(F_{K}\right)$ between two distinct vertices if and only if there are representatives of the corresponding isotopy classes which are disjoint. $\Gamma\left(F_{K}\right)$ is called the arc complex of $F_{K}$ (see [Masur and Minsky 1999], for example).

Now, $F_{K}$ divides $M_{K}$ into two submanifolds, $H$ and $H^{\prime}$. Let $V$ and $V^{\prime}$ denote the sets of vertices of $\Gamma\left(F_{K}\right)$ corresponding to curves that bound compressions in $H$ and $H^{\prime}$, respectively. Then $d(K, F)$, the distance of $K$ with respect to $F$, is defined to be the number of edges in the shortest path from $V$ to $V^{\prime}$ in $\Gamma\left(F_{K}\right)$. As long as $\chi\left(F_{K}\right)$ is at most -2 this is well defined, since the arc complex is connected in those cases.

\section{Lemmas}

The following is a slight variant of the Haken Lemma [1968]. We assume familiarity with the proof of this result found in [Jaco 1980, Theorem II.7]. 
Lemma 4.1 (Haken). Let $K$ be a knot in a 3-manifold $M$ which is in bridge position with respect to a Heegaard surface $F$. If $M_{K}$ contains an essential 2-sphere or meridional disk then $d(K, F)=0$.

Proof. Among all essential 2-spheres and meridional disks in $M_{K}$ choose one, $S$, meeting $F_{K}$ minimally. Let $H$ and $H^{\prime}$ denote the submanifolds of $M_{K}$ bounded by $F_{K}$, with $\partial S$ (if nonempty) contained in $H$. If $S \cap F_{K}=\varnothing$ then $S$ lies entirely in $H$ or $H^{\prime}$, a contradiction. It follows that $S \cap F_{K}$ is a nonempty set of loops that are essential on $F_{K}$. Hence, if $S$ meets $F_{K}$ in a single loop, the result follows.

Suppose then that $\left|S \cap F_{K}\right|>1$. Let $H^{*}$ denote one of $H$ or $H^{\prime}$, where there is a component $T$ of $S \cap H^{*}$ with $|\partial T-\partial S| \geq 2$. Choose a basis $\Lambda$ for $H^{*}$, that is, a system of disks and bigons cutting $H^{*}$ into a 3-ball. If $S \cap \Lambda$ contains any loops, surger $S$ along these loops, innermost (on $\Lambda$ ) first. At least one component of the resulting surface is again an essential sphere or meridional disk. We continue to denote this surface by $S$.

Now reduce $|S \cap \Lambda|$ as follows. If any component of $\left(S \cap H^{*}\right)-\Lambda$ is a bigon, surger $\Lambda$ along this surface. Some subcollection of the resulting set is again a basis, which we continue to denote by $\Lambda$. If not, choose a bigon of $\Lambda-S$, and use this to guide an isotopy of $S$ (see the "isotopy of type A" in [Jaco 1980, p. 24]). Repeat this procedure until all components $T$ of $S \cap H^{*}$ satisfy $|\partial T-\partial S|=1$. Let $S^{\prime}$ denote the resulting surface.

It follows from the argument of [Jaco 1980, Lemma II.9] that if $H^{*}=H^{\prime}$ then $\left|S^{\prime} \cap F_{K}\right|<\left|S \cap F_{K}\right|$, and we have reached a contradiction. If $H^{*}=H$ then $\left|S^{\prime} \cap F_{K}\right| \leq\left|S \cap F_{K}\right|$. If equality holds we repeat the preceding steps with $S^{\prime}$ replacing $S$ and letting $H^{*}=H^{\prime}$. This gives a surface $S^{\prime \prime}$ with $\left|S^{\prime \prime} \cap F_{K}\right|<\left|S \cap F_{K}\right|$, a contradiction.

Lemma 4.2. Let $K$ be a knot in a 3-manifold $M$ which is in bridge position with respect to a Heegaard surface $F$. Suppose $\gamma$ bounds two cut surfaces $A$ and $B$ with $A \cap B=\gamma$. Then $A$ and $B$ are both bigons, both annuli, or both disks, unless $d(K, F)=0$.

Proof. If $A$ and $B$ are of different types, their union is a meridional disk. The result now follows from Lemma 4.1.

Lemma 4.3. Let $K$ be a knot in a 3-manifold $M$ which is in bridge position with respect to a Heegaard surface $F$ and let $Q$ be any properly embedded surface in $M_{K}$. If there is a curve $\gamma$ that is essential on $Q$ and bounds a cut surface $E$ in $M_{K}$ then either there is a curve $\gamma^{\prime} \subset E \cap Q$ that bounds a compression for $Q$, unless $d(K, F)=0$.

Proof. Let $\Lambda \subset E \cap Q$ be the collection of curves that are essential on $Q$. Let $E^{\prime}$ denote the closure of a component of $E-\Lambda$ that is a cut surface. Set $\gamma^{\prime}=E^{\prime} \cap \Lambda$. 
Consider the set $\Theta$ of cut surfaces bounded by $\gamma^{\prime}$ such that the only curve of intersection with $Q$, essential on $Q$, is $\gamma^{\prime}$. Note that $E^{\prime}$ is such a surface, so $\Theta$ is nonempty. Let $E^{*}$ be an element of $\Theta$ with $\left|E^{*} \cap Q\right|$ minimal.

We now claim $E^{*} \cap Q=\gamma^{\prime}$. Suppose not. Let $E^{\prime \prime}$ be a cut surface component of $E^{*}-Q$. The curve $\gamma^{\prime \prime}=E^{\prime \prime} \cap Q$ is inessential on $Q$ and hence bounds two cut surfaces, $A \subset Q$ and $E^{\prime \prime}$. Note that $A \cap E^{\prime \prime}=\gamma^{\prime \prime}$. By Lemma 4.2 we may obtain a new cut surface from $E^{*}$ by replacing $E^{\prime \prime}$ with a push-off of $A$. This violates the minimality of $\left|E^{*} \cap Q\right|$. We conclude that $E^{*}$ is a compression for $Q$, which finishes the proof.

Lemma 4.4. Let $K$ be a knot in a 3-manifold $M$ which is in bridge position with respect to a Heegaard surface $F$ and let $S$ be an essential surface in $M_{K}$. If we surger $S$ along a disk or bigon cut surface then at least one of the remaining components is essential, unless $d(K, F)=0$.

Proof. By assumption there is a curve $\gamma \subset S$ that bounds a cut surface $E^{\prime}$, homeomorphic to a disk and such that $E^{\prime} \cap S=\gamma$. Since $S$ is essential, $\gamma$ bounds a cut surface $E \subset S$. Surgering $S$ along $E^{\prime}$ produces two surfaces, isotopic to $E \cup E^{\prime}$ and $S^{\prime}=(S-E) \cup E^{\prime}$. Suppose $S^{\prime}$ is not essential. Let $\gamma^{\prime}$ bound a compression $C$ for $S^{\prime}$. As $E^{\prime}$ is homeomorphic to a disk we may properly isotope $\gamma^{\prime}$ off of $E^{\prime}$. The curve $\gamma^{\prime}$ is now on $S$, and bounds the cut surface $C$. By Lemma 4.3 there is a compression $C^{\prime}$ for $S$, a contradiction.

Lemma 4.5. Let $K$ be a knot in a 3-manifold $M$ which is in bridge position with respect to a Heegaard surface $F$ and let $S$ be an essential surface in $M_{K}$. If we surger $S$ along a cut surface then at least one of the remaining components is essential, unless $d(K, F)=0$.

Proof. By assumption there is a curve $\gamma \subset S$ which bounds a cut surface $E^{\prime}$ such that $E^{\prime} \cap S=\gamma$. Since $S$ is essential, $\gamma$ bounds a cut surface $E$ in $S$. Surgering $S$ along $E^{\prime}$ then produces two surfaces, isotopic to $E \cup E^{\prime}$ and $S^{\prime}=(S-E) \cup E^{\prime}$.

By Lemma 4.4 we may assume $E^{\prime}$ is an annulus. By Lemma 4.2 we may assume $E$ is also an annulus. If $E \cup E^{\prime}$ is essential, we are done. Otherwise there must be a compressing bigon $B$ for $E \cup E^{\prime}$ (since the core loop of $E \cup E^{\prime}$ is not essential). Surgering $E \cup E^{\prime}$ along $B$ gives a disk $D$ with $\partial D \subset \partial M_{K}$ bounding a disk $D^{\prime} \subset$ $\partial M_{K}$. If the sphere $D \cup D^{\prime}$ is essential, the proof is complete by Lemma 4.1. Otherwise we conclude that $E \cup E^{\prime}$, together with an annulus of $\partial M_{K}$, bounds a solid torus. If the interior of the solid torus is disjoint from $S$ then $S^{\prime}$ is properly isotopic to $S$ and we are done. If $S$ meets the interior of the solid torus we may push it entirely into the solid torus. Now consider $B \cap S$. Some component of $B-S$ is then a cut surface for $S$. This cut surface is either a disk or a bigon. By Lemma 4.4 we may surger $S$ along this cut surface and obtain another essential surface that meets $B$ fewer times. Continuing in this way we obtain an essential 
surface inside the solid torus that misses $B$, and is hence contained in a ball. This is impossible.

\section{Proof of the Main Theorem}

We recall the statement.

Theorem 5.1. Let $K$ be a knot in a closed, orientable 3-manifold $M$ which is in bridge position with respect to a Heegaard surface $F$. Let $S$ be a properly embedded, orientable, essential surface in $M_{K}$. Then the distance of $K$ with respect to $F$ is bounded above by twice the genus of $S$ plus $|\partial S|$.

We now begin the proof. Throughout we assume that $d(K, F)>0$ to avoid the special cases of the lemmas from Section 4 . Let $\Sigma_{0}$ and $\Sigma_{1}$ denote spines of the handlebodies bounded by $F$. Let $h: M \rightarrow I$ denote a height function on $M$ such that $h^{-1}(0)=\Sigma_{0}$ and $h^{-1}(1)=\Sigma_{1}$. We require that for every $t \in(0,1)$ the surface $h^{-1}(t)$ is parallel to $F=h^{-1}\left(\frac{1}{2}\right)$. Because $K$ is in bridge position with respect to $F$ we may isotope $K$ so that each arc of $K-F$ has one critical point with respect to $h$. Now pull each minimum down to $\Sigma_{0}$ and each maximum up to $\Sigma_{1}$. If $M=S^{3}$ and $F$ is a sphere we may assume that $K$ has at least two maxima and at least two minima. In this case $\Sigma_{0}$ and $\Sigma_{1}$ are edges, and we assume that the vertices $\partial \Sigma_{0}$ coincide with two minima of $K$ and the vertices $\partial \Sigma_{1}$ coincide with two maxima.

Set $F(t)=h^{-1}(t) \cap M_{K}$. Let $H(t)$ be the closure of the component of $M_{K}-$ $F(t)$ that contains $\Sigma_{0}$. Let $H^{\prime}(t)$ be the closure of $M_{K}-H(t)$. Let $\epsilon_{0}$ be chosen just larger than the radius of $N(K)$, but small enough so that $S$ meets $H\left(\epsilon_{0}\right)$ and $H^{\prime}\left(1-\epsilon_{0}\right)$ in compressions for $F\left(\epsilon_{0}\right)$ and $F\left(1-\epsilon_{0}\right)$. Then the surface $F(t)$ is homeomorphic to $F_{K}=F \cap M_{K}$ for every value of $t \in\left[\epsilon_{0}, 1-\epsilon_{0}\right]$. Hence, the submanifold $\bigcup_{t=\epsilon_{0}}^{1-\epsilon_{0}} F(t)$ is homeomorphic to $F_{K} \times\left[\epsilon_{0}, 1-\epsilon_{0}\right]$. Let $\pi$ denote the composition of such a homeomorphism with projection onto the first factor. Hence, if $\gamma$ is a curve on $F(t)$ for some $t \in\left[\epsilon_{0}, 1-\epsilon_{0}\right]$, then $\pi(\gamma)$ is a curve on $F_{K}$.

We make two types of assumptions on the position of the essential surface $S$. Any surface whose position satisfies these assumptions we will say is in standard position. The first concerns how $S$ meets $\partial M_{K}$ and the second is a genericity assumption on the interior of $S$. Near the boundary of $S$ we assume the following:

- Meridional boundary components are "level"; that is, if $S$ has meridional boundary, there exists for each boundary component $C$ of $S$ a $t \in\left(\epsilon_{0}, 1-\epsilon_{0}\right)$ such that $C \subset \partial F(t)$. We consider $t$ a critical value for $S$ if some boundary component of $S$ is contained in $\partial F(t)$.

- If $S$ does not have meridional boundary then for generic $t$ and each component $\gamma$ of $\partial S-F(t)$ the endpoints of $\gamma$ lie on distinct boundary components of $F(t)$. 


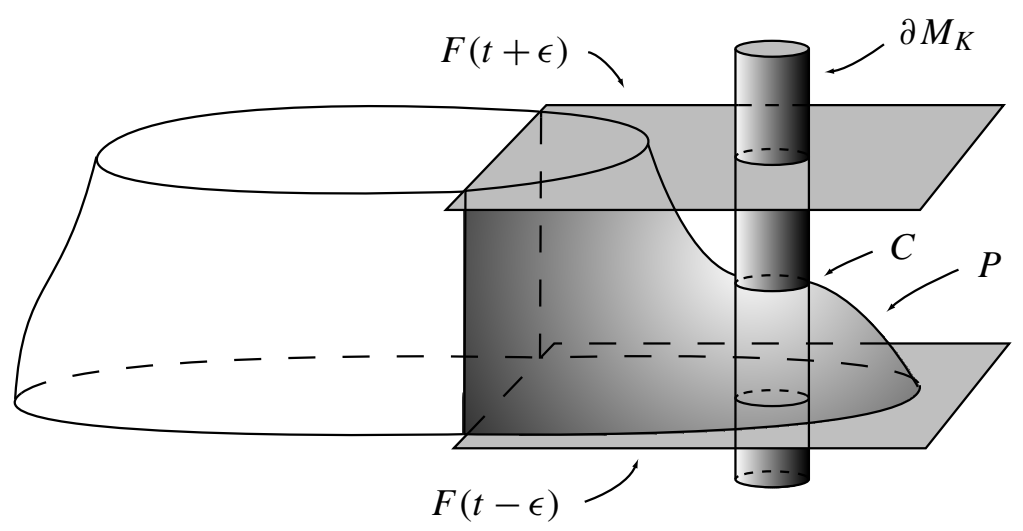

Figure 2. A piece of $S$ between levels, $F(t-\epsilon)$ and $F(t+\epsilon)$, before and after a meridional boundary component, $C$.

These stipulations are possible since $\partial S$ is not null-homotopic on $\partial M_{K}$. In the interior of $M_{K}$ we assume the position of $S$ is generic in the following sense:

- All critical points of $\left.h\right|_{S}$ are maxima, minima, or saddles. We will refer to any such critical point whose height is between $\epsilon_{0}$ and $1-\epsilon_{0}$ and to any meridional boundary component as a critical submanifold (of $S$ ).

- The heights of any two critical submanifolds of $S$ are distinct.

- Suppose a meridional boundary component $C$ of $S$ happens at height $t$. Let $P$ denote the closure of the component of $S-F(t \pm \epsilon)$ that has $C$ as a boundary component. Then $P$ is a once-punctured annulus with one boundary component on each of $F(t-\epsilon)$ and $F(t+\epsilon)$ (see Figure 2). (This uses the fact that $\partial M_{K}$ is connected.)

Claim 5.2. For each $t \in\left[\epsilon_{0}, 1-\epsilon_{0}\right]$ the submanifolds $H(t)$ and $H^{\prime}(t)$ of $M_{K} d o$ not contain any essential surfaces.

Proof. Choose a basis $\Lambda$ of compressing disks and bigons in $H(t)$ that cut it into a ball. Suppose $D \in \Lambda$. Let $D^{\prime}$ be a cut surface component of $D-Q$, where $Q$ is some essential surface in $H(t)$. By Lemma 4.4, compressing $Q$ along $D^{\prime}$ yields an essential surface that meets $D$ fewer times. Continuing in this way we produce an essential surface in $H(t)$ disjoint from $\Lambda$, and hence in a ball. This is impossible.

Definition 5.3. Let $t_{0}$ be the supremum of $t \in\left[\epsilon_{0}, 1-\epsilon_{0}\right]$ such that some curve in $S \cap F(t)$ bounds a compression for $F(t)$ in $H(t)$. (The compression for $F(t)$ need not be a subsurface of $S$.) Define $t_{1}$ likewise with infimum instead of supremum and $H^{\prime}(t)$ instead of $H(t)$. 
Claim 5.4. The values $t_{0}$ and $t_{1}$ are well defined, and $t_{0}>\epsilon_{0}$.

Proof. To establish the claim it is enough to show that for some small $\epsilon>\epsilon_{0}$ there are curves in $S \cap F(\epsilon)$ and $S \cap F(1-\epsilon)$ that bound compressions for $F(\epsilon)$ and $F(1-\epsilon)$ in $H(\epsilon)$ and $H^{\prime}(1-\epsilon)$, respectively.

There are essentially two cases. Suppose first the essential surface $S$ is closed, or has meridional boundary. If $S \cap \Sigma_{0}=\varnothing$ then $S$ can be properly isotoped entirely into $H^{\prime}(\epsilon)$, violating Claim 5.2. We conclude that $S \cap \Sigma_{0} \neq \varnothing . \quad F(\epsilon) \cap S$ then contains a loop that bounds a compression for $F(\epsilon)$ in $H(\epsilon)$. On the other hand, if $S$ has nonempty, nonmeridional boundary then $F(\epsilon) \cap S$ contains an arc that bounds a bigon compression in $H(\epsilon)$. This proves that $t_{0}$ is well defined and $t_{0}>\epsilon>\epsilon_{0}$. A symmetric argument shows $t_{1}$ is well defined.

Claim 5.5. The value of $t_{0}$ is less than $1-\epsilon_{0}$.

Proof. Suppose $t_{0}=1-\epsilon_{0}$. Let $\epsilon>\epsilon_{0}$ be small enough that $1-\epsilon$ is greater than the height of the highest critical submanifold. As $t_{0}=1-\epsilon_{0}$ there is a curve $\alpha$ of $F(1-\epsilon) \cap S$ that is essential in $F(1-\epsilon)$ but bounds a compression in $H(1-\epsilon)$.

Recall that the boundary of $S$ has been isotoped into standard position. It follows that the components of $S \cap H^{\prime}(1-\epsilon)$ are all disks and bigons. Hence, $\alpha$ bounds compressions for $F(1-\epsilon)$ on both sides and $d(K, F)=0$.

Claim 5.6. If $t_{0}=t_{1}<1-\epsilon_{0}$ then $d(K, F)=1$.

Proof. If $t_{0}=t_{1}<1-\epsilon_{0}$ then for all sufficiently small $\epsilon$ there is a curve of $S \cap F\left(t_{0}+\epsilon\right)$ bounding a compression in $H^{\prime}(t)$ and a curve of $S \cap F\left(t_{0}-\epsilon\right)$ bounding a compression in $H(t)$. But for $\epsilon$ sufficiently small the curves of $\pi\left(S \cap F\left(t_{0}+\epsilon\right)\right)$ can be made disjoint from the curves of $\pi\left(S \cap F\left(t_{0}-\epsilon\right)\right)$, because $F$ and $S$ are orientable. This is basically identical to [Gabai 1987, Lemma 4.4].

Henceforth we assume that $\epsilon_{0}<t_{0}<t_{1}<1-\epsilon_{0}$.

Claim 5.7. If $t_{*} \in\left(t_{0}, t_{1}\right)$ is a critical value then for sufficiently small $\epsilon$ the curves of $\pi\left(F\left(t_{*}-\epsilon\right) \cap S\right)$ are at a distance of at most one from the curves of $\pi\left(F\left(t_{*}+\epsilon\right) \cap S\right)$.

Proof. As in the proof of Claim 5.6, the curves of $\pi\left(S \cap F\left(t_{*}+\epsilon\right)\right)$, for $\epsilon$ sufficiently small, can be made disjoint from the curves of $\pi\left(S \cap F\left(t_{*}-\epsilon\right)\right)$. The result follows unless either of these are collections of inessential curves, and hence are not represented in $\Gamma\left(F_{K}\right)$. However, if this is the case then all curves of $S \cap F\left(t_{*}+\epsilon\right)$ (say) are inessential on $S$. By Lemma 4.5 a sequence of surgeries produces an essential surface disjoint from $F\left(t_{*}+\epsilon\right)$, contradicting Claim 5.2.

Claim 5.8. A component of $F(t) \cap S$ that is inessential on $F(t)$ is inessential on $S$.

Proof. This follows directly from Lemma 4.3. 
Now let $t \in\left[\epsilon_{0}, 1-\epsilon_{0}\right]$ be a regular value of $\left.h\right|_{S}$. Pick a component $\gamma$ of $F(t) \cap S$. The curve $\gamma$ is mutually essential if it is essential on both $F(t)$ and $S$, mutually inessential if it is inessential on both and mutual if it is mutually essential or mutually inessential. Finally, $\gamma$ is special if it is inessential on $S$ but essential on $F(t)$. There are three kinds of special curves: loops that bound disks on $S$, loops that cobound (with $\partial S$ ) annuli in $S$, and arcs isotopic (via bigons) into $\partial S$.

Claim 5.9. Suppose $t$ is a regular value of $\left.h\right|_{S}$ in $\left[t_{0}, t_{1}\right]$. Every curve of $F(t) \cap S$ is mutual.

Proof. Pick a regular value $t \in\left[\epsilon_{0}, 1-\epsilon_{0}\right]$. By Claim 5.8 we may assume that there is a special curve $\gamma$ in $F(t) \cap S$. By definition, $\gamma$ is essential on $F(t)$ but inessential on $S$. It follows that a component $E$ of $S-\gamma$ is a cut surface. By Lemma 4.3 there is a curve of $E \cap F(t)$ that bounds a compression for $F(t)$. This compression either lies in $H(t)$ or in $H^{\prime}(t)$. Since $E \cap F(t) \subset S \cap F(t)$ we conclude $t \notin\left[t_{0}, t_{1}\right]$.

Claim 5.10. If $\alpha$ is an arc component of $F(t) \cap S$ and $h(\alpha)=t \in\left(t_{0}, t_{1}\right)$ then $\alpha$ is mutually essential.

Proof. By Claim 5.9 the only other possibility is that $\alpha$ is mutually inessential. In this case $\partial \alpha$ is the boundary of some arc $\gamma$ of $\partial S-F(t)$. Also, $\partial \gamma=\partial \alpha$ lies on the same component of $\partial F(t)$. This violates our assumption that $S$ is in standard position.

In $h^{-1}\left(\left[t_{0}, t_{1}\right]\right)$ we see the usual four types of critical submanifolds for $S$ : maxima, minima, saddles, and meridional boundary components. Suppose a critical submanifold happening at height $t$ is a saddle or meridional boundary component. Let $P$ be the closure of the component of $S-F(t \pm \epsilon)$ that contains the critical submanifold. We call $P$ a horizontal neighborhood (in $S$ ) of the critical submanifold. Let $\partial_{ \pm} P=P \cap F(t \pm \epsilon)$. We say the critical submanifold at $t$ is special if there is some component of $\partial_{ \pm} P$ that is special. If the critical submanifold at $t$ is not special, we say it is inessential if some component of the closure of $S-P$ is a disk and essential otherwise. If the critical submanifold at $t$ is inessential, Claim 5.10 implies that there is a mutually inessential loop component of $\partial_{ \pm} P$ that bounds a disk in $S$.

Claim 5.11. Suppose $t_{*} \in\left[t_{0}, t_{1}\right]$. If there is a special critical submanifold at $t_{*}$ then $t_{*}=t_{0}$ or $t_{1}$.

Proof. By definition, if a special critical submanifold happens at $t_{*}$ there is a special curve $\alpha$ in $S \cap F\left(t_{*}-\epsilon\right)$ or $S \cap F\left(t_{*}+\epsilon\right)$. Assuming the former, Claim 5.9 implies $t_{*}-\epsilon \notin\left[t_{0}, t_{1}\right]$. Hence $t_{*}=t_{0}$. If, on the other hand, $\alpha \subset F\left(t_{*}+\epsilon\right)$, we deduce $t_{*}=t_{1}$. 

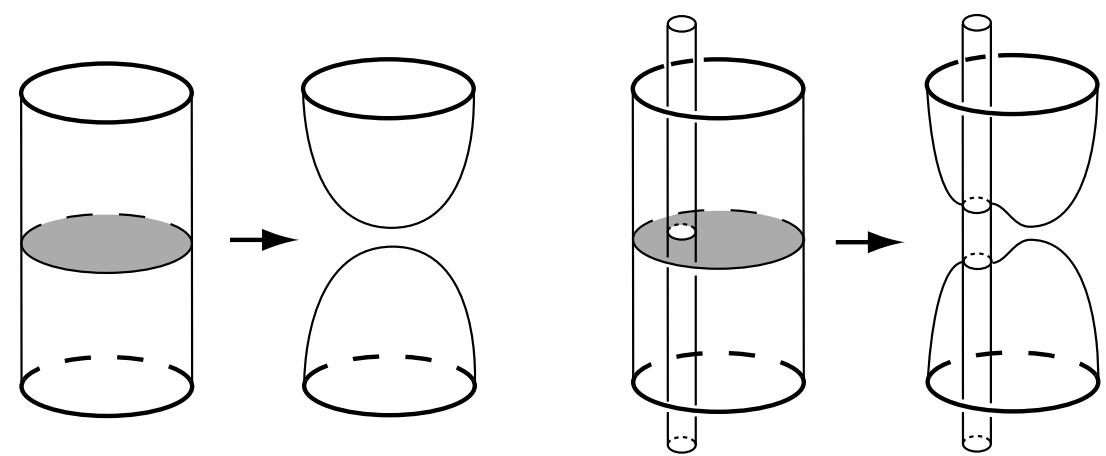

Figure 3. Constructing $S^{\prime}$ from $S$. On the left two new critical values are created. On the right four are created.

Lemma 5.12. Let $t_{-}$and $t_{+}$be regular values in $\left[t_{0}, t_{1}\right]$ such that every saddle and every meridional boundary component of $S$ in $h^{-1}\left(t_{-}, t_{+}\right)$is inessential. Then $\pi\left(F\left(t_{-}\right) \cap S\right)$ and $\pi\left(F\left(t_{+}\right) \cap S\right)$ share a vertex in $\Gamma\left(F_{K}\right)$.

Proof. Let $\left\{t_{i}\right\}$ be the critical values of $\left.h\right|_{S}$ lying in $\left[t_{-}, t_{+}\right]$. Choose $r_{i}$ slightly greater than the $t_{i}$ and let $R=\left\{r_{i}\right\} \cup\left\{t_{-}+\epsilon\right\}$.

For every $r \in R$ surger $S$ in the following way. If $S \cap F(r)$ contains mutually inessential curves, some such curve bounds a cut surface in $F(r)$. Surger $S$ along this cut surface. After a sequence of such surgeries we obtain from $S$ a surface that meets $F(r)$ only in mutually essential curves, for all $r \in R$.

Set $M^{\prime}=h^{-1}\left(\left[t_{-}, t_{+}\right]\right)$. Let $S^{\prime}$ be the intersection of the surgered surface with $M^{\prime}$. Note that $\left.h\right|_{S^{\prime}}$, the height function restricted to $S^{\prime}$, has either two or four new critical values for every surgery performed. See Figure 3.

We say a surface $V$ is vertical in $M^{\prime}$ if $V=\pi^{-1}(\alpha) \cap M^{\prime}$, where $\alpha$ is a properly embedded one-manifold in $F_{K}$. A vertical surface $V$ is either a disk or an annulus. We need the following claim to prove the lemma:

Claim 5.13. Each component $S^{\prime \prime}$ of $S^{\prime}$ is either

- a sphere or a meridional annulus, or

- properly isotopic into $F\left(t_{-}\right)$or $F\left(t_{+}\right)$, or

- properly isotopic to a vertical surface $V$ with $\pi(V)$ essential in $F_{K}$.

Proof. If $\left.h\right|_{S^{\prime \prime}}$ has no critical values, $S^{\prime \prime}$ is isotopic to a vertical annulus or disk. In this case $S^{\prime \prime} \cap \partial M^{\prime}$ must be essential by the construction of $S^{\prime}$. Note that this kind of situation is the desired conclusion of the lemma at hand. If $\left.h\right|_{S^{\prime \prime}}$ has only critical values of even index (and no meridional boundary components) then $S^{\prime \prime}$ is a boundary parallel disk or a sphere. 
We now assume that $S^{\prime \prime}$ contains a critical submanifold which is not a max or min. The component $S^{\prime \prime}$ either contains a saddle or meridional boundary component of $S$, or it does not. Suppose the latter. It follows that $S^{\prime \prime}$ is either a meridional annulus or a boundary parallel annulus (with one boundary component on $\partial M_{K}$ ).

Now suppose that $S^{\prime \prime}$ contains a saddle or meridional boundary component of $S$ at height $t_{*}$. Let $P$ be the closure of the component of $S^{\prime \prime}-F\left(t_{*} \pm \epsilon\right)$ that contains this critical submanifold. (Note that $P$ is also a subsurface of $S$ since $\epsilon$ is very small.) Recall that $P$ is the horizontal neighborhood of the critical submanifold. Let $\partial_{ \pm} P=P \cap F\left(t_{*} \pm \epsilon\right)$. Since every critical submanifold of $S \cap M^{\prime}$ is inessential, at least one loop component of $\partial_{ \pm} P$ bounds a disk in $S$ (see the comment preceding Claim 5.11).

Now suppose that $S^{\prime \prime}$ contains a meridional boundary component of $S$ at height $t_{*}$. Let $P$ be the corresponding horizontal neighborhood. Let $\partial_{ \pm} P=C_{1} \cup C_{2}$, where $C_{1}$ bounds a disk $D$ in $S$. Hence, $D \cup P \subset S$ is a cut annulus and we see that $C_{2}$ is also inessential in $S$. By Claim 5.9 the $C_{i}$ are inessential in $F\left(t_{*} \pm \epsilon\right)$. It now follows from Lemma 4.2 that $C_{1}$ bounds a disk in $F\left(t_{*} \pm \epsilon\right)$ while $C_{2}$ bounds a cut annulus in $F\left(t_{*} \mp \epsilon\right)$. Thus $S^{\prime \prime}$ is a meridional annulus.

We now assume that $S^{\prime \prime}$ contains no meridional boundary components of $S$, and hence contains a saddle. Suppose some such saddle has a horizontal neighborhood $P$ such that two components of $\partial_{ \pm} P$ are inessential. It follows that all three components are inessential. If two bound disks, all three do. Therefore, by Lemma 4.2, $S^{\prime \prime}$ is a sphere. If one bounds a disk and the other two bound cut annuli then $S^{\prime \prime}$ is a meridional annulus.

Finally, we assume that $S^{\prime \prime}$ contains no meridional boundary components and that every saddle $x$ has a horizontal neighborhood $P_{x}$ with exactly one component $\gamma_{x}$ of $\partial_{ \pm} P_{x}$ inessential, bounding a disk in $S$ (see Figure 4). By Claim 5.9 and Lemma 4.2 it follows that $\gamma_{x}$ bounds a disk in $S^{\prime \prime}$. Hence $S^{\prime \prime}$ is either a union of disks or a union of annuli. In the first case $S^{\prime \prime}$ is isotopic to a vertical disk. In
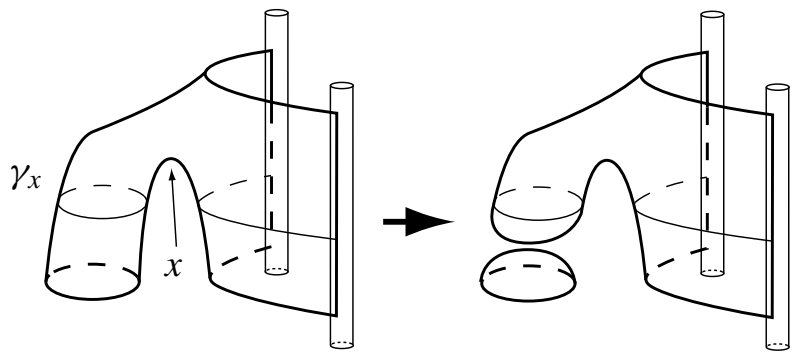

Figure 4. Surgery near a saddle whose horizontal neighborhood has exactly one inessential boundary component. 
the latter case $S^{\prime \prime}$ is either isotopic to a vertical annulus or is a boundary parallel annulus.

To complete the proof of the Lemma 5.12, suppose no component of $S^{\prime}$ meets both boundary components of $M^{\prime}$. By Claim 5.13, every component of $S^{\prime}$ meeting $F\left(t_{-}\right)$is boundary parallel in $M^{\prime}$. Isotope $F\left(t_{-}\right)$across these boundary parallelisms to obtain a surface $F^{\prime}$ that intersects the surface $S$ only in mutually inessential curves. Some component of $F^{\prime}-S$ is then a cut surface, which we may use to surger $S$. By Lemma 4.5 we obtain an essential surface that meets $F^{\prime}$ in fewer curves. Continuing in this fashion we obtain an essential surface disjoint from $F^{\prime}$, violating Claim 5.2.

We conclude that there is a component $S^{\prime \prime} \subset S^{\prime}$ meeting both $F\left(t_{-}\right)$and $F\left(t_{+}\right)$. By Claim 5.13, this $S^{\prime \prime}$ must be isotopic to a vertical annulus or vertical disk with essential boundary. The lemma is thus proved.

We now complete the proof of Theorem 5.1. Note that when $t \in\left[t_{0}, t_{1}\right]$ is a regular value, $\pi(F(t) \cap S)$ is a properly embedded 1-manifold in $F_{K}$ (recall that $\left.F_{K}=F \cap M_{K}\right)$. The distance between the loops and arcs of $\pi\left(F\left(t_{0}-\epsilon\right) \cap S\right)$ and of $\pi\left(F\left(t_{1}+\epsilon\right) \cap S\right)$ in $\Gamma\left(F_{K}\right)$ is an upper bound for the distance $d(K, F)$. By Lemma 5.12 and Claim 5.7 this number is bounded by the number of essential critical submanifolds, $e$, plus the number of special critical submanifolds. By Claim 5.11 this latter number is at most two. We therefore conclude $d(K, F) \leq e+2$.

We now bound the Euler characteristic of $S$. Suppose an essential critical submanifold happens at $t_{*}$ and let $P$ be its horizontal neighborhood in $S$. Note that in all cases $\chi(P)=-1$. (When $P$ has vertical boundary compute its Euler characteristic by doubling across the vertical boundary and taking half of the Euler characteristic of the resulting surface. See, for example, the surface on the left in Figure 4.) By the definition of an essential critical submanifold $\partial P-\partial S$ is essential in $S$. We conclude that $\chi(S) \leq-e$.

Putting these facts together we conclude that

$$
d(K, F) \leq e+2 \leq-\chi(S)+2=-(2-2 g(S)-|\partial S|)+2=2 g(S)+|\partial S| .
$$

\section{Applications}

We now present a few quick corollaries to Theorem 5.1.

Corollary 6.1. Suppose $K$ is a knot in $S^{3}$ whose distance is $d(K, F)$ with respect to a bridge sphere $F$. Then the genus of $K$ is at least $\frac{1}{2}(d(K, F)-1)$.

Proof. The genus of $K$ is defined to be the smallest genus of all orientable spanning surfaces for $K$. Such a spanning surface is essential and has exactly one boundary component. Hence, an immediate application of Theorem 5.1 implies $d(K, F) \leq$ $2 g(K)+1$. 
Corollary 6.2. If $K$ is a knot whose distance is at least 3 with respect to some Heegaard surface, the complement of $K$ is hyperbolic of finite volume.

Proof. If the distance is greater than two, $M_{K}$ is irreducible, atoroidal, anannular, and has incompressible boundary. It follows from Thurston's geometrization theorem for Haken manifolds that $M_{K}$ is hyperbolic of finite volume.

Definition 6.3. Suppose $M$ is obtained by removing a neighborhood of a knot $K$ in $S^{3}$ and gluing in a new solid torus to the resulting boundary component. Then we say that $M$ was obtained by Dehn surgery on $K$.

Corollary 6.4. Suppose $K$ is a knot in $S^{3}$ whose distance is $d(K, F)$ with respect to a bridge sphere $F$. If a manifold $M$ obtained by Dehn surgery on $K$ contains an incompressible torus $T$, then $\left|\partial\left(T \cap M_{K}\right)\right|$ is at least $d(K, F)-2$.

Proof. Choose $T$ so as to minimize $|T \cap K|$ in $M$. Let $T_{K}=T \cap M_{K}$. follows from the minimality assumption that $T_{K}$ is essential. Theorem 5.1 says that $d(K, F)$ is bounded above by twice the genus of $T_{K}$ plus $\left|\partial T_{K}\right|$. But $T$ is a torus, so the genus of $T_{K}$ is one.

Corollary 6.5. Suppose $K$ is a knot in $S^{3}$ whose distance with respect to some bridge sphere is greater than its bridge number. Then a minimal bridge presentation for $K$ is thin.

Proof. Let $F$ be a bridge sphere for which $d(K, F) \geq|K \cap F|$. If thin position for $K$ does not equal bridge position then by [Thompson 1997] there is a planar, meridional, essential surface $S$ in the complement of $K$ with fewer boundary components than $|K \cap F|$. Hence, by Theorem 5.1 the distance $d(K, F)$ is at most $|\partial S| \leq|K \cap F|$.

\section{References}

[Akiyoshi et al. 2000] H. Akiyoshi, M. Sakuma, M. Wada, and Y. Yamashita, "Ford domains of punctured torus groups and two-bridge knot groups", Sūrikaisekikenkyūsho Kōkyūroku (=Suri Kaiseki Kenkyujo kokyuroku) 1163 (2000), 67-77. MR 1799369 Zbl 0969.57505

[Gabai 1987] D. Gabai, "Foliations and the topology of 3-manifolds, III", J. Differential Geom. 26:3 (1987), 479-536. MR 89a:57014b Zbl 0639.57008

[Haken 1968] W. Haken, "Some results on surfaces in 3-manifolds", pp. 39-98 in Studies in Modern Topology, Math. Assoc. Amer., Buffalo and Prentice-Hall, Englewood Cliffs (NJ), 1968. MR 36 \#7118 Zbl 0194.24902

[Hartshorn 1999] K. Hartshorn, Heegaard splittings: the distance complex and the stabilization conjecture, Ph.D. thesis, University of California, Berkeley, 1999.

[Hempel 2001] J. Hempel, "3-manifolds as viewed from the curve complex", Topology 40:3 (2001), 631-657. MR 2002f:57044 Zbl 0985.57014

[Jaco 1980] W. Jaco, Lectures on three-manifold topology, CBMS Regional Conference Series in Mathematics 43, Amer. Math. Soc., Providence, 1980. MR 81k:57009 Zbl 0433.57001 
[Kobayashi 1988] T. Kobayashi, "Heights of simple loops and pseudo-Anosov homeomorphisms", pp. 327-338 in Braids (Santa Cruz, CA, 1986), edited by J. S. Birman and A. Libgober, Contemp. Math. 78, Amer. Math. Soc., Providence, RI, 1988. MR 89m:57015 Zbl 0663.57010

[Masur and Minsky 1999] H. A. Masur and Y. N. Minsky, "Geometry of the complex of curves, I: Hyperbolicity”, Invent. Math. 138:1 (1999), 103-149. MR 2000i:57027 Zbl 0941.32012

[Morgan and Bass 1984] J. Morgan and H. Bass (editors), The Smith conjecture (New York, 1979), edited by J. Morgan and H. Bass, Pure and Applied Mathematics 112, Academic Press, Orlando, FL, 1984. MR 86i:57002 Zbl 0599.57001

[Morimoto 1989] K. Morimoto, "On minimum genus Heegaard splittings of some orientable closed 3-manifolds", Tokyo J. Math. 12:2 (1989), 321-355. MR 91b:57020 Zbl 0714.57007

[Morimoto and Sakuma 1991] K. Morimoto and M. Sakuma, "On unknotting tunnels for knots", Math. Ann. 289:1 (1991), 143-167. MR 92e:57015 Zbl 0697.57002

[Saito 2004] T. Saito, "Genus one 1-bridge knots as viewed from the curve complex", Osaka J. Math. 41:2 (2004), 427-454. MR 2069094 Zbl 02111464

[Thompson 1997] A. Thompson, "Thin position and bridge number for knots in the 3-sphere", Topology 36:2 (1997), 505-507. MR 97m:57013 Zbl 0867.57009

Received August 29, 2003. Revised October 2, 2003.

DAVID BACHMAN

Pitzer College

1050 Mills AVE

Claremont, CA 91711

bachman@pitzer.edu

SAUL SCHLEIMER

Mathematics Department

UNIVERSITY OF ILLINOIS AT CHICAGO

Chicago, IL 60607

saul@math.uic.edu 